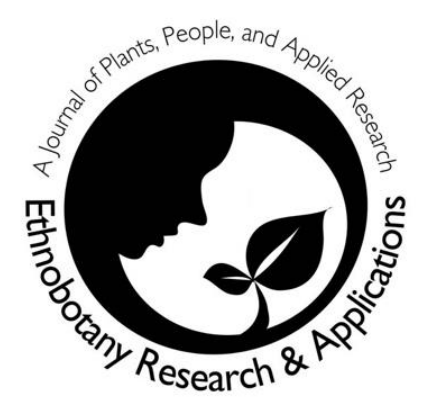

\title{
Diversity and Ethnobotany of genus Garcinia L. (Clusiaceae) in Assam, Eastern Himalaya
}

\author{
Sanjib Baruah, Pranjan Barman, Sanswrang \\ Basumatary, Birina Bhuyan
}

\section{Databases and Inventories}

\begin{abstract}
The genus Garcinia is an important component of the flora of Assam and is well known for being used traditionally in many different ways. To preserve this knowledge, this study recorded the ethnobotanical importance of the genus Garcinia in this region. The study revealed that there are 12 species and one variety of the genus indigenous to Assam.
\end{abstract}

Methods: This study was conducted in different parts of Assam, Northeast India based on extensive field work involving collection and documentation of members of genus Garcinia. Important characteristic features of the specimens like colour of leaf and stem, branching pattern of the stems, habit, habitat, size, etc. were noted down in the field during collection. Ethnobotanical data were collected by interviewing local communities through a semi-structured questionnaire, interview and literature review.

Results: In present communication, we provide ethnobotanical and botanical descriptions and illustrations of 12 Garcinia species that are popularly used among ethnic communities of Assam for fruits, medicines and fixative or as a mordant for saffron dye. These species consist of G. anomala Planch. \& Triana., G. assamica J. Sarma, Shameer \& N. Mohanan, G. cowa Roxb. Ex Choisy, G. dulcis (Roxb.) Kurz, G. indica (Thouars) Choisy, G. kydia Roxb., G. lanceifolia Roxb., G. morella (Gaertn.) Desr., G. paniculata Roxb. ex Wight, G. pedunculata Roxb. Ex Buch-Ham., G. nervosa Miq. And G. xanthocymus Hook.f. ex T.Anderson.

Conclusions: This study concludes that documentation and preservation of traditional knowledge associated with Garcinia could generate further research activities for the benefit of human kind. The traditional utilization of Garcinia members observed are most prevalent amongst the rural communities of the studied area. It is also a fact that in spite of having ample economic potential, the species of the genus Garcinia occurring in Assam and also in northeast India has not been studied properly.

\section{Correspondence}

Sanjib Baruah ${ }^{1^{*}}$, Pranjan Barman ${ }^{2}$, Sanswrang Basumatary ${ }^{1}$, Birina Bhuyan ${ }^{1}$

${ }^{1}$ Department of Botany, Bodoland University, Bodoland territorial Region (BTR), Assam 783370, India.

2Department of Biotechnology, Gauhati University, Assam - 781014, India

"Corresponding Author: Sanjib Baruah; sanjibbaruah9@gmail.com

Ethnobotany Research \& Applications

21:33 (2021)

Key words: Garcinia, Clusiaceae, Diversity,

Ethnobotany, Assam, Eastern Himalaya

\section{Background}

Assam is one of the Indian states of Eastern Himalayas recognized as a hotspot of biodiversity, possessing an enormous plant wealth. Geographically, Northeast India is a part of Southeast Asia. The state is not only rich in biodiversity but also has the distinction of having rich ethnic diversity and rich flora and fauna (Baruah et al. 2018). A total of 4273 species comprising of 1448 genera, distributed in 272 families of vascular plants were recorded from Assam, which represents about $25.12 \%$ of the total 
flora of India (Chowdhury 2005). But unfortunately, there are areas and certain plant groups still remain to be explored scientifically. Assam is very rich in ethnic diversity and has a great indigenous knowledge base in plant resources. It has more than 21 major tribes and 35 other sub tribes with their own indigenous traditional knowledge and great cultural practices adapting with the forest biodiversity (Baruah et al. 2012).

The genus Garcinia L. belongs to the family Clusiaceae and comprises of about 250 species in world. Garcinia members are distributed in Malaysian region to South Asia ranging from southern parts of Thailand and Indonesia but mainly confined to South East Asian region (Mabberly 2005, Sharma et al.1993). In peninsular Malaysia there are 49 Garcinia species out of 250 species estimated worldwide (Stevens 2007, Whitemore 1973). The name Garcinia honors a French botanist, Laurent Garcin (1683-1751) who lived and worked in India. In India the genus hosts by 43 species and 5 varieties, of which 37 species and 4 varieties occur in wild, whereas 6 species and 1 variety introduced into cultivation (Anderson 1874, Maheshwari 1964, Mohanan et al. 1997, Sabu et al. 2013, Singh 1993, Srivastava 1994). In Northeast India, it is represented by 17 species- of which 2 species and 1 variety are endemic to the region. In flora of Assam, Kanjilal et al. (1934) reported 9 species from undivided Assam. Kar et al. (2008) reported 8 species from Sonitpur districts of Assam. In addition to the above, the occurrence of certain taxa was reported for the first time from Assam viz., G. indica (Sarma et al. 2014) G. dulcis (Begam et al. 2013) G. nervosa (Dutta et al. 2014). Recently, Sharma et al. (2016) discovered Garcinia assamica from Manas National Park and surroundings, Assam. Garcinia species are considered as taxonomically complicated as majority are dioecious in nature and complex in floral characters. However, several useful identification keys have been reported for Garcinia species across the globe based on morphological features of flower, fruit and leaf (Jones 1980, Nimanthika \& Kaththriarachchi 2010). This present study was conducted to highlight the diversity and the ethnobotanical knowledge associated with the Garcinia species reported by the local population and herbalists in Assam.

\section{Materials and methods \\ Study area}

Assam is located at the central part of the NorthEast India and with an area of $78,438 \mathrm{~km}^{2}$ representing $2.39 \%$ of Indian land mass situated in between $24018 /$ and $28018 / \mathrm{N}$ latitude to $89042 /$ and 96o 30/E longitude (Baruah et al. 2011). The state is bordered on the east by Nagaland, Manipur and Myanmar, on the west by West Bengal, on the north by Bhutan and Arunachal Pradesh and on the south by Meghalaya, Bangladesh, Tripura and Mizoram. Topographically Assam can be divided to two major divisions, i.e., the plains and the hills. The plains can further be divided into two physiographic divisions: (i) the Brahmaputra valley and (ii) the Barak valley. The hills are also divided into two units - the Karbi Plateau and North Cachar Hills (Barooah \& Sarma 2016). The locations of the present study area are covers both the Brahmaputra valley and Barak valley lies between $240 \mathrm{18} /$ and $280 \mathrm{18} / \mathrm{N}$ latitude to $890 \mathrm{0} / \mathrm{2}$ and 960 30/E longitude (Figure 1).

\section{Taxonomy}

The present study is based on field work involving collection and documentation of members of genus Garcinia made regularly from different districts of Assam. Important characteristic features of the specimens like colour of leaf and stem, branching pattern of the stems, habit, habitat, size, etc. were noted down in the field during collection. Provisional identification of the specimens was made by comparing the field notes and observations with the descriptions available in authentic literatures. The specimens on which the present study is based have been preserved following standard herbarium techniques (Jain \& Rao 1977). The nomenclature was confirmed by consulting herbarium sheets in India (ARUN, ASSAM and CAL) and foreign digital herbaria $(A, M O, N Y$ ) (acronyms following Thiers 2018). The voucher specimens are deposited in the Bodoland University Herbarium (BUH), Kokrajhar, Assam. The distribution map of all collected species in the study area has been prepared by using ArcGIS 10.5.

\section{Ethnobotanical study}

Field work relevant to the ethnobotanical information was undertaken during the period of January 2018 to February 2020. Field survey was carried out by interviews with local people, elderly people as well as traditional healers of the study site having knowledge of folk medicine and plants. Interview technique employed based on questionnaire to collect the data about the use of Garcinia members. The interviews were conducted with 45 men and 40 women from different parts of Assam reputed for ethnobotanical knowledge and expertise. Among the informants, 20 claimed to be traditional healers. The age group of informants ranged from 20 to 75 years. Apart from this, secondary information on the traditional knowledge uses of different species of Garcinia was collected from different sources like journals, articles, floras etc. 


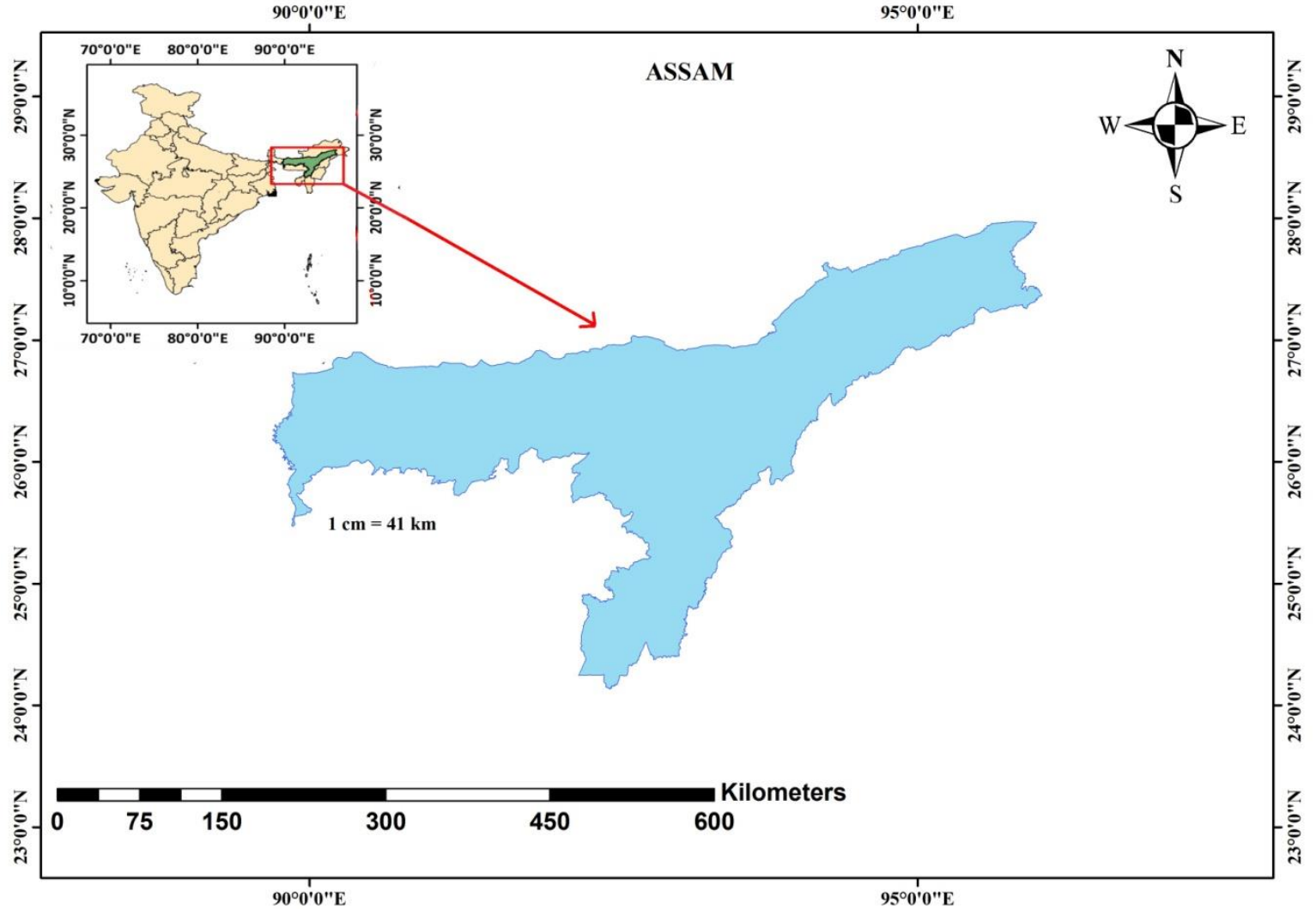

Fig. 1. MAP showing the study area

\section{Results}

\section{Species Descriptions and Distributions}

A total of 12 species and 1 variety were recorded from the present political boundary of Assam. The detail distributions of recorded Garcinia members in the state of Assam is showing in Figure 2.

1. Garcinia anomala Planch. \&Triana (Figure 3) Dioecious; evergreen trees, Bark exudes yellowish. Leaves simple, opposite, decussate; lamina elliptic or oblong-lanceolate 9-20 $\times 4-9 \mathrm{~cm}$, green, round at base, acuminate at apex. Flowers polygamous; Male flowers 12-20 mm., pale green; pedicels ca. 2$4 \mathrm{~mm}$ long; bracts 2; bracteoles 2, ca. 1.5-3.5 mm long, opposite; sepals 4, ca. 8-10 mm long, petals 4, ca. 10-12 mm long, yellow; stamens indefinite; filaments short, free, compressed. Female flowers: sepals persistent; petals whitish-green; ovary bilocular, oblong, locules 1-ovuled; stigma disciform. Berries ca. $3.5-4.5 \mathrm{~cm}$, ellipsoid, orangeyellow when ripe. Seeds 1-2.
Phenology:-Flowering February-May, fruiting August.

Distribution: India (Assam: Manas National Park)

2. Garcinia assamica J. Sarma, Shameer \& N. Mohanan (Figure 4)

Dioecious; evergreen trees 15-20 m tall, branchlets terete; bark latex greenish-yellow; Leaves leathery, elliptic-oblong, $\quad 6-10.5 \times 2-5.5 \mathrm{~cm}$, apex acuminate; lateral veins $10-12$ pairs; petioles $1-1.5$ $\mathrm{cm}$ long, angular. Flowers grouped in axillary or terminal fascicles. Male flowers 1-3 creamy-white; pedicel 4-7 mm long. Sepals 4, orbicular, ca. $6 \times 8$ $\mathrm{mm}$. Petals 4, pale white, 6-9 × 4-6 mm. Stamens ca. 22-25. Female flowers $2-5$ at each node, creamy-white. Sepals 4 , orbicular, ca. $6 \times 6 \mathrm{~mm}$, membranous. Petals 4, 8-10 × 5-8 mm, pale yellow. Staminodes 4-5, slender, brownish. Ovary globose 4-5-locular, vertically grooved; stigmatic rays $8-10$. Fruit turbinate, ca. $7 \times 5 \mathrm{~cm}$, distinct mammilla, greenish turning orange-yellow when ripe. Seeds 2-5. 


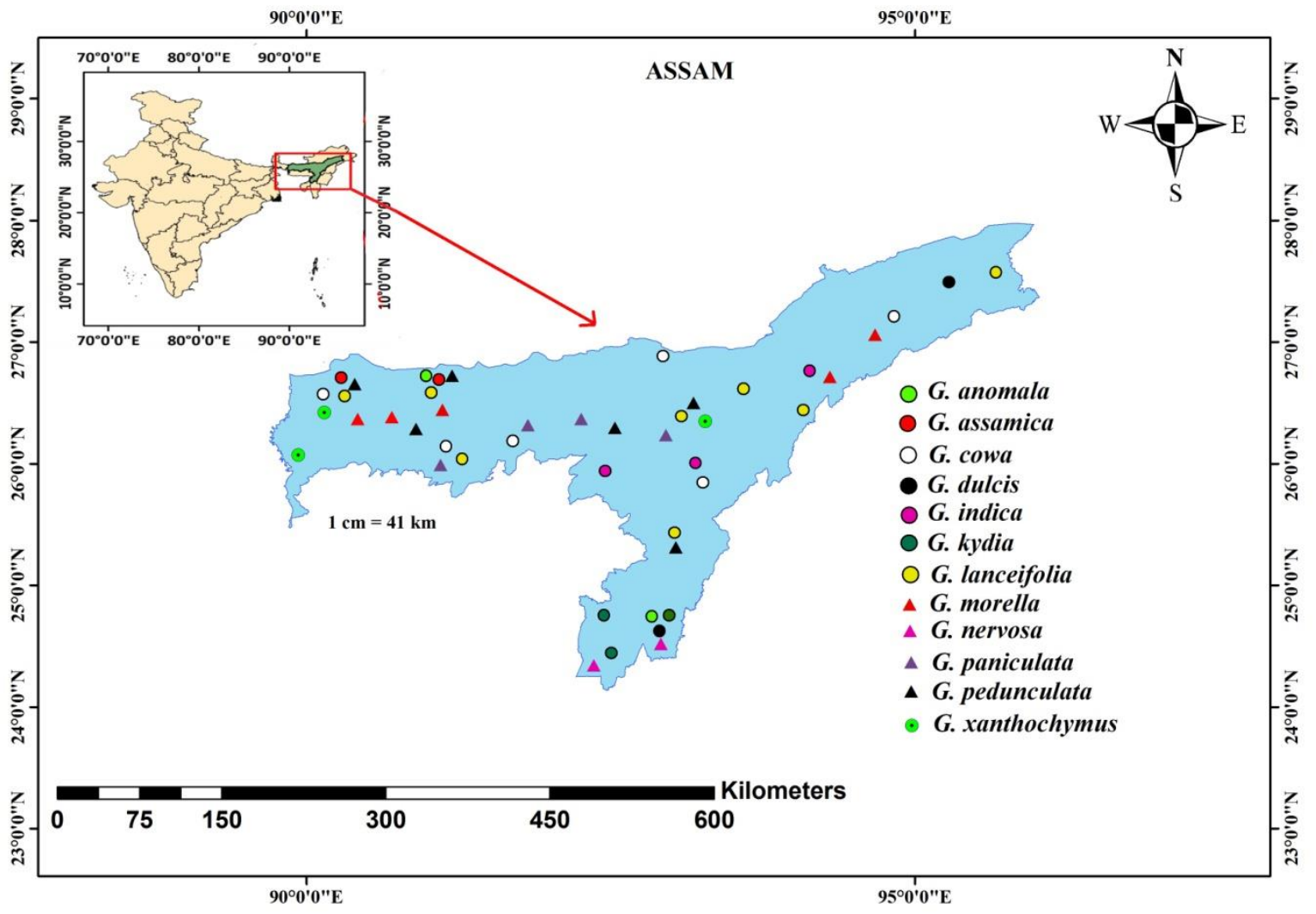

Fig. 2. The map above shows distribution of Garcinia members in Assam

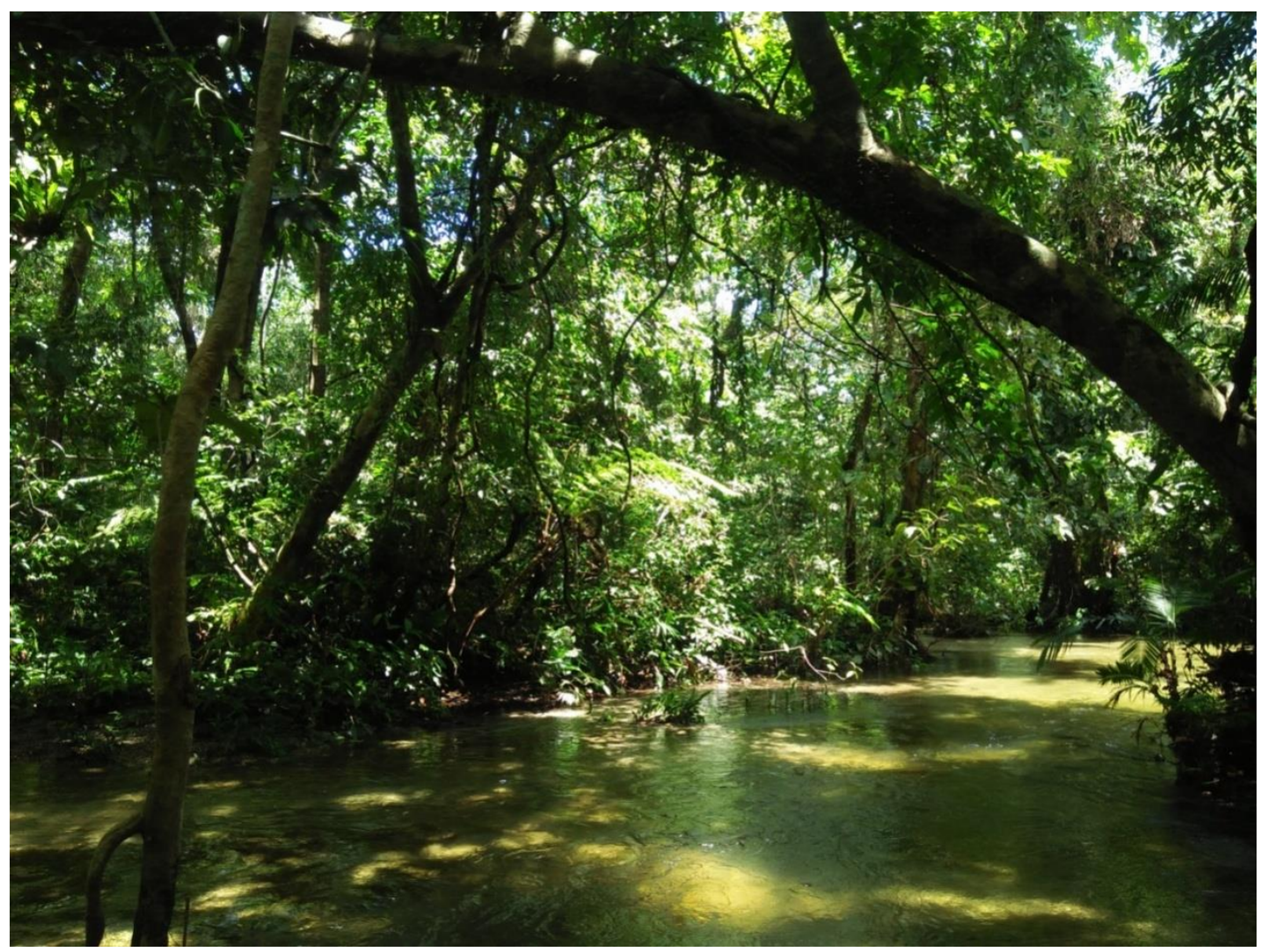

Fig. 3. G. anomala in natural forest of Assam (Photo: Sanjib Baruah) 


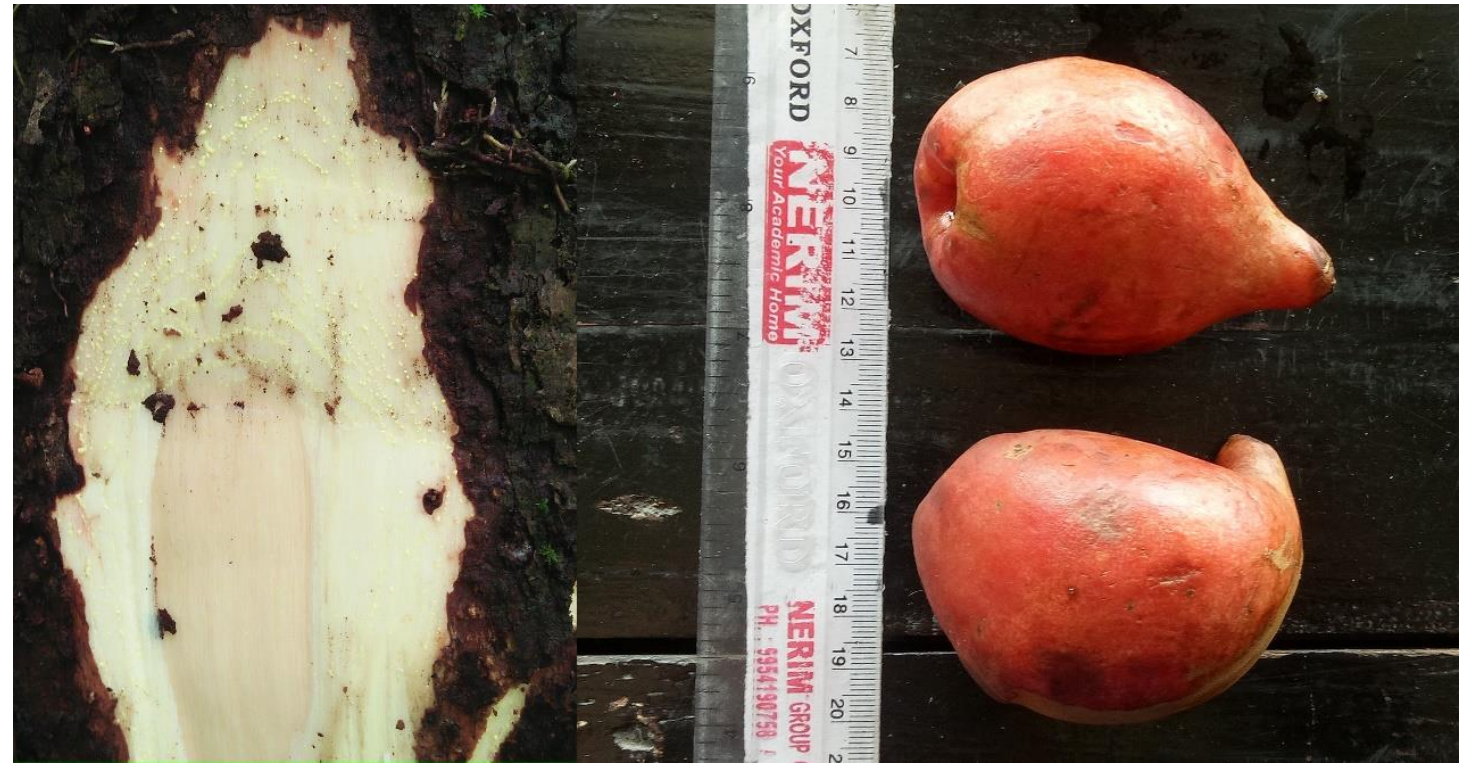

Fig. 4. Garcinia assamica (Photo: Sanjib Baruah)

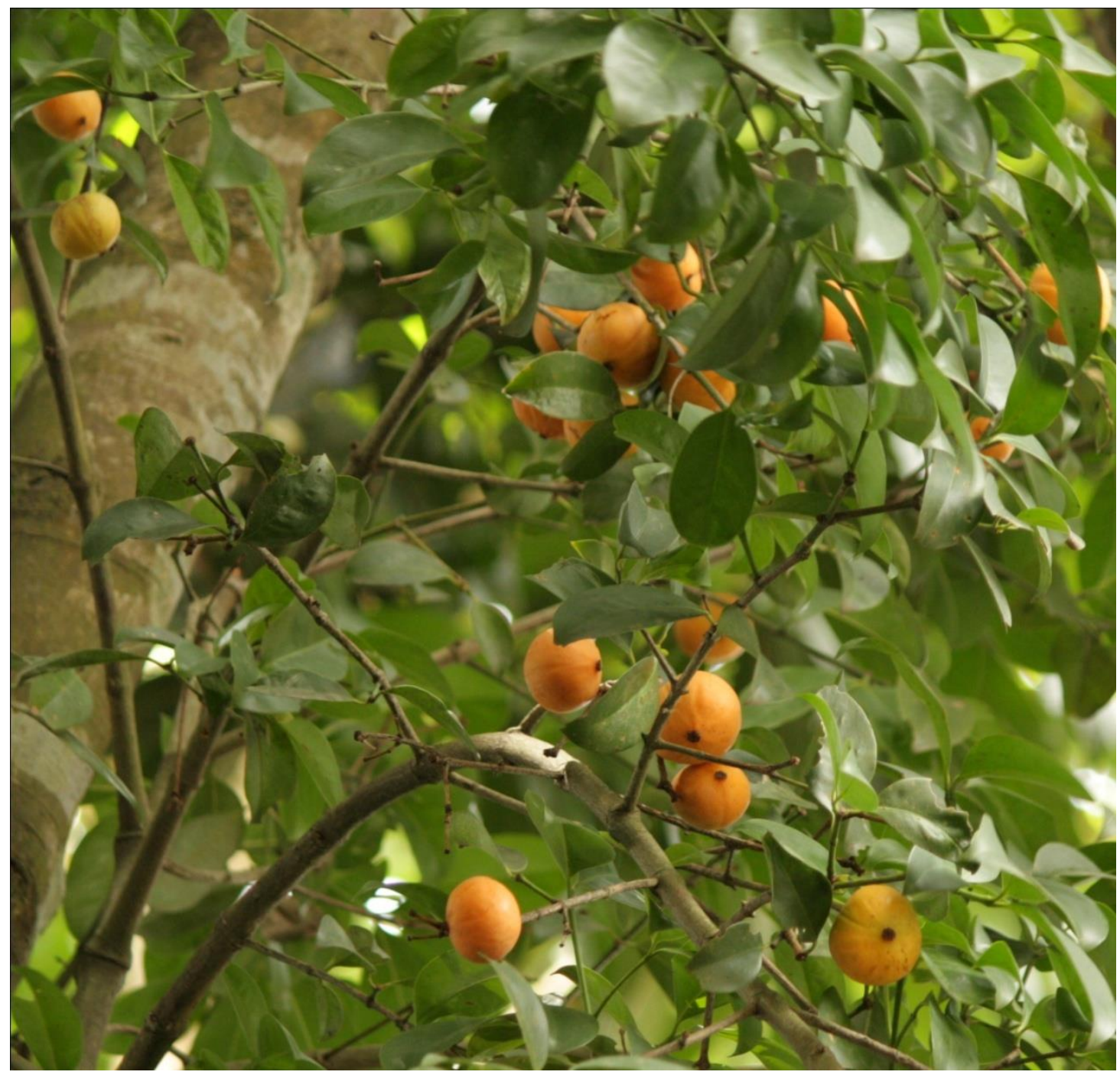

Fig. 5. Garcinia cowa (Photo: Jatindra Sarma) 
3. Garcinia cowa Roxb. ex Choisy (Figure 5)

Dioecious; evergreen middle sized trees. Bark smooth; latex creamy yellowish, sticky. Leaves simple, opposite, decussate, 7-14 × 2.5-7 cm; lamina 8-16 $\times$ 2.5-6 $\mathrm{cm}$, elliptic-oblong, oblanceolate; midvein prominent, lateral vein not prominent. Flowers polygamous, Male flowers: 1 $\mathrm{cm}$ across, 3-8 in axillary or terminal fascicles; pedicels ca. $6 \mathrm{~mm}$ long; sepals 4, 4-6 mm long, broadly ovate, fleshy, yellow, stamens numerous. Female flowers: $1.5 \mathrm{~cm}$ across, $2-5$ in terminal fascicles, yellow; pedicel short; ovary superior, subglobose, 6-8 locular, ovules one in each cell; stigma sessile. Fruit a berry, $2-4 \mathrm{~cm}$ across, globose, oblique towards to the apex, smooth, yellow. Seeds 4-8, oblong.

Phenology:-Flowering March-April, Fruiting JuneAugust.

Distribution: Assam and Meghalaya.

\section{Garcinia dulcis (Roxb.) Kurz (Figure 6)}

Dioecious; evergreen trees, bark dark blackish colored, latex thick yellowish. Leaves simple opposite, elliptic-oblong to ovate; lamina coriaceous, elliptic, obovate, oblong-lanceolate, acute or obtuse, base obtuse, $9-30 \times 4-15 \mathrm{~cm}$; mid-vein prominent beneath. Flowers, $2.5-4 \mathrm{~cm}$ long. Male flowers: yellow, stamens in 5 bundles or 4 bundles, connate at the base, filaments short, anthers peltate, oblong, 2 loculed. Female flowers: globose, with minute staminodes, filaments arranged in ring shape, ovary superior, globose, 5 locular, ovule 1 per locule, style short, thick, stigma peltate, rays 5 , margins rotundate. Fruit fleshy berry, globose, $2.5-3.5 \times 1.5-2.5 \mathrm{~cm}$ across, yellow. Seeds 1-5, oblong-ovoid, yellow pulp.

Phenology:-Flowering April-May, Fruiting JulyNovember

Distribution: India (Assam, Andaman \& Nicobar Islands), Thailand, Java, Borneo and the Philippines.

\section{Garcinia indica (Thouars) Choisy (Figure 7)}

Dioecious; middle size trees, to 12 - $15 \mathrm{~m}$ height. Leaves simple, opposite, decussate, estipulate; petiole $5-12 \mathrm{~mm}$ long; lamina $6.5-12 \times 1.5-4 \mathrm{~cm}$, lanceolate or obovate-oblong, base attenuate, apex acute to acuminate, Flowers polygamodioecious; Male flowers: 4-8 in axillary and terminal fascicles; sepals 4, yellowish-orange to pinkish-orange, coriaceous, ovate- rotundate, outer ones $3-4.5 \mathrm{~mm}$ long, inner ones 4.5-5 mm long; petals 4, 5-6 mm long, thick; stamens many; filaments short; anthers oblong, truncate, loculi laterally introrse; rudimentary pistil absent or a few; female flowers: solitary, terminal; pedicels $3 \mathrm{~mm}$ long; sepals and petals as in male flowers; staminodes $10-18$, in 4 unequal, 2 to 3 seriate phalanges alternating with petals, 1-3 mm long; ovary superior, 4-8 locular, subglobose; stigma 4-8 rayed, convex, coronate, rays tuberculate, often 2 -seriate. Fruit berry, $2-4 \mathrm{~cm}$ across, 4-8 loculed, purple or wine brown, surrounded by persistent calyx; pulp whitish red; seeds 5-8, acidic pulp.

Phenology:-Flowering \& Fruiting November-April Distribution: India (Assam, Andaman \& Nicobar Islands), Thailand, Java, Borneo and the Philippines.

\section{Garcia kydia Roxb.}

Dioecious; evergreen trees, up to $18-20 \mathrm{~m}$ tall, narrow crowned; wood white-yellowish; Bark blackish brown, latex yellowish; Branchlets glabrous, often drooping. Leaves simple, opposite, decussate; petioles 8-12 mm long; lamina 8.8-15 $\times$ $2.5-4.5 \mathrm{~cm}$, ovate-oblong rarely obovate-oblong, apex acuminate. Male flowers: 2-3, axillary, sometimes solitary; peduncles 8-15 mm long; pedicels 5-6 mm long; sepals 4, yellow, equal, ovate, obtuse, fleshy; petals 4, 9-12 mm long, pale yellow, broadly ovate, blunt, thick, concave; anthers squarish, bilocular, 4-lobed, filaments conjoined. Female flowers solitary, axillary and terminal; sepals 4, 4-6 mm long, yellow, ovate, obtuse, fleshy; petals 4, 8-12mm long, yellow; staminodes 4, small; ovary 6-8 lobed, globular, 6-8-locular; stigmas subsessile. Berries $2.5-5.5 \mathrm{~cm}$, depressed, with a nipple-like protuberance from apex, dark purple-brown. Seeds 6-8, oblong.

Phenology:-Flowering \& Fruiting November-April Distribution: India (Assam, Andaman \& Nicobar Islands, West Bengal), Malaysia, Myanmar.

\section{Garcinia lanceifolia Roxb. (Figure 8)}

Dioecious; small evergreen trees, wood hard, bark dark brown, latex yellowish. Leaves simple, opposite, lanceolate, ca. 5-12 × 2-3.5 cm across, attenuate base, margins entire, apex acuminate to cuspidate, glabrous on both sides, midrib prominent beneath, lateral veins 8-18, irregular; petiole slender, stout, about $0.5-1.5 \mathrm{~cm}$ long, exstipulate. Inflorescence axillary solitary; Flowers polygamous, 4 merous. Male flowers 1-2 terminal, solitary, dark, bracteate, sepals 4, imbricate, oblong, yellowish green, petals 4 , imbricate, narrow obovate, slightly oblique, yellow, stamens many, filaments short, anthers oblong, 2 loculed. Female flowers: axillary, solitary, sepals 4 , ovate, membranous, petals 4 , narrow, ovary superior, globose, 6-20 locular. Seeds 6-8, oblong.

Phenology:-Flowering February-March; Fruiting June-July

Distribution: India (Assam, Meghalaya \& Nagaland), Malaysia, Myanmar 


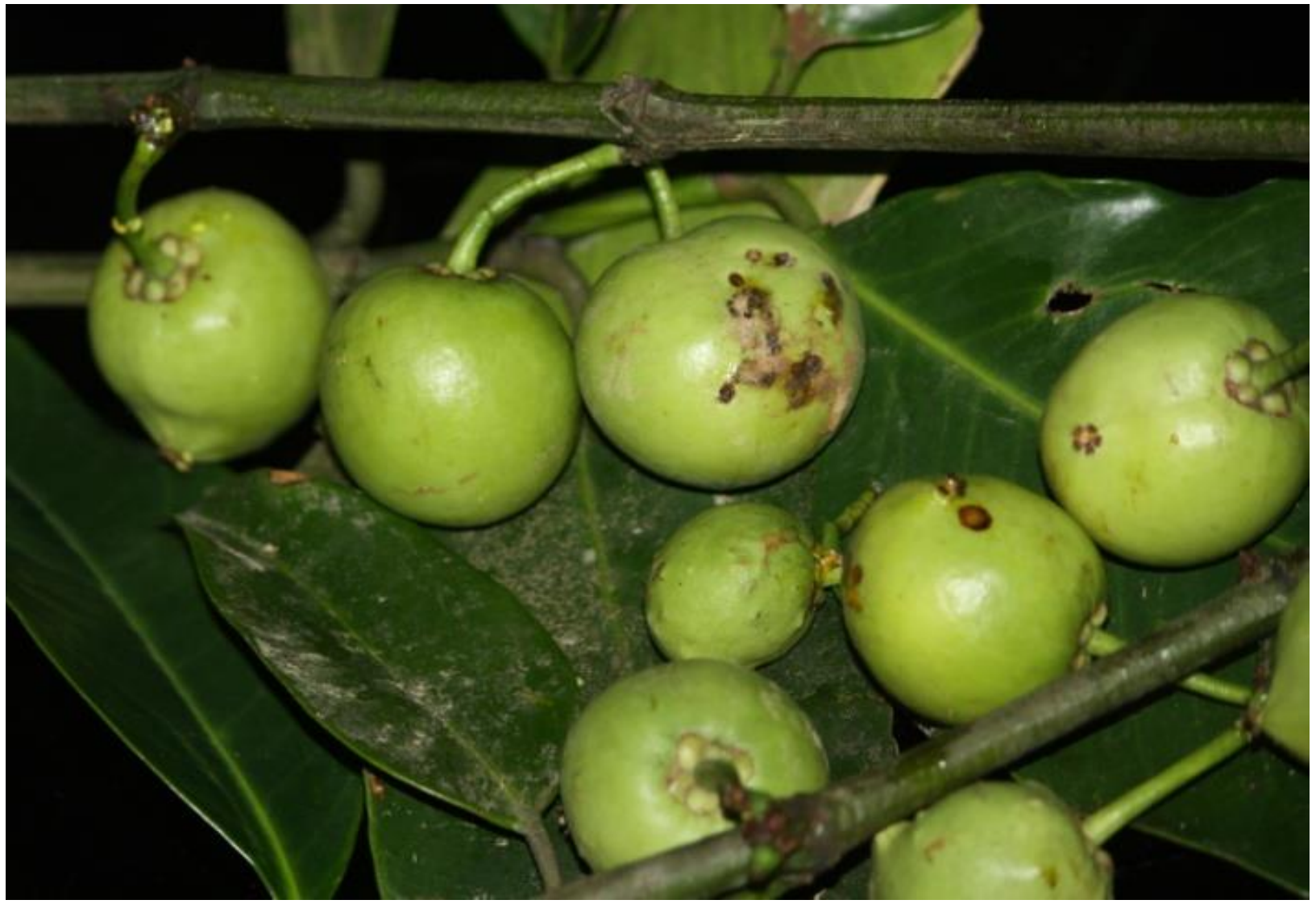

Fig. 6. Garcinia dulcis (Photo: Jatindra Sarma)

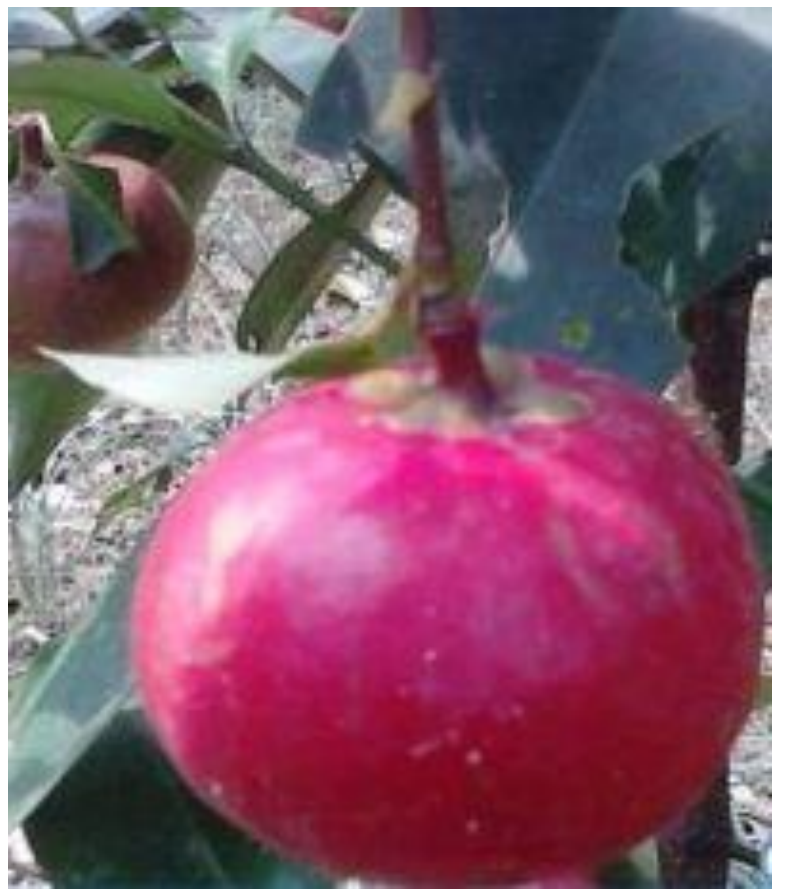

Fig. 7. Fruits of Garcinia indica (Photo: Sanjib Baruah)

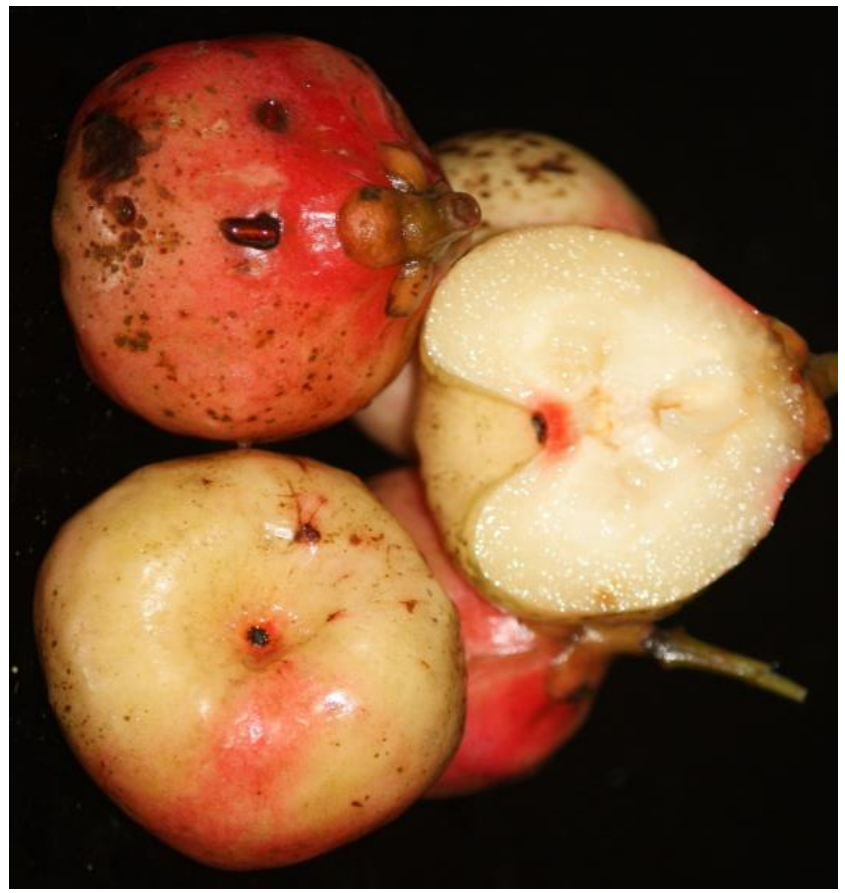

Fig. 8. Garcinia lanceifolia (Photo:Jantindra Sarma) 


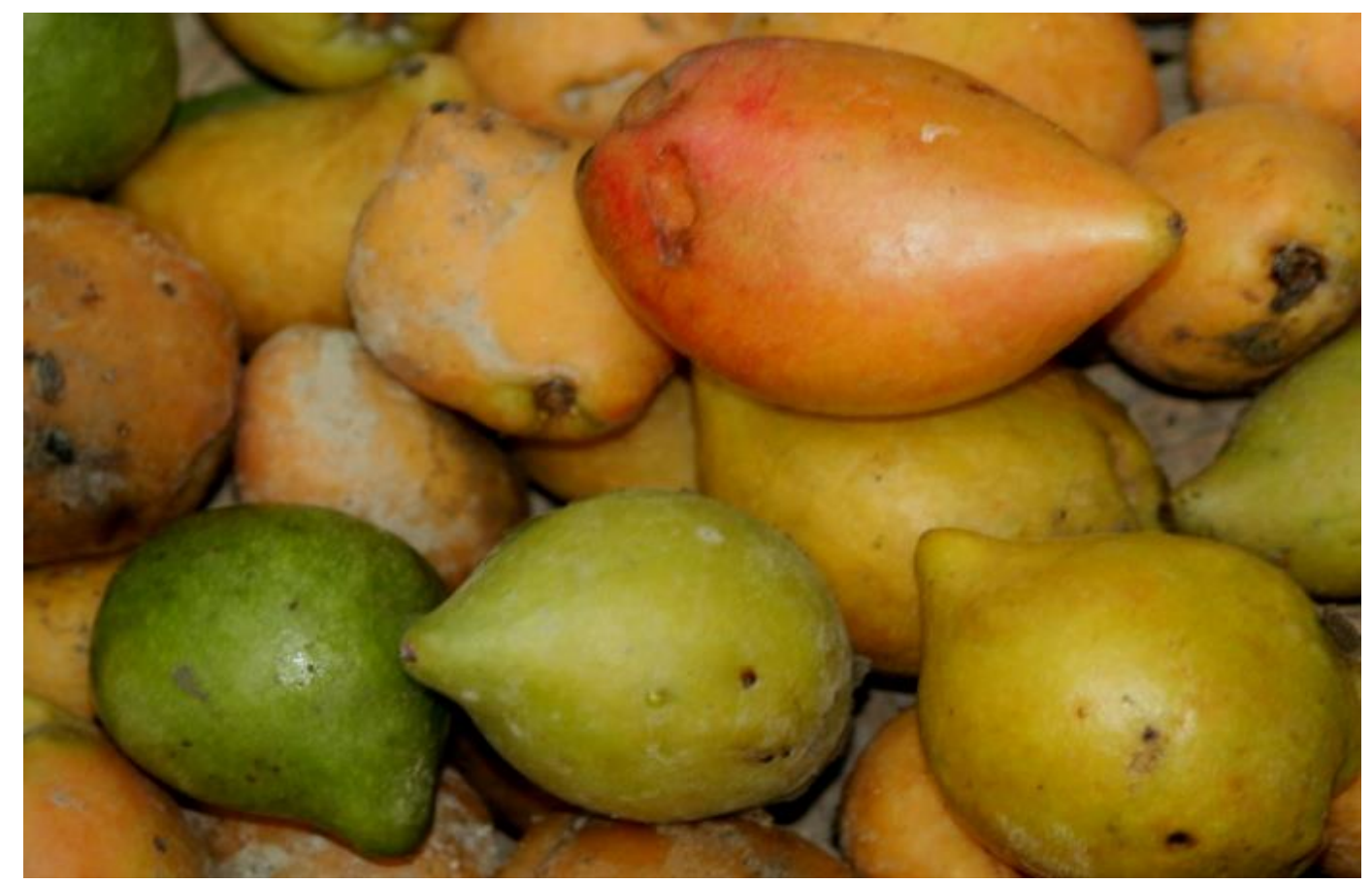

Fig. 9. Mature fruits of Garcinia morella (Photo: Jatindra Sarma)

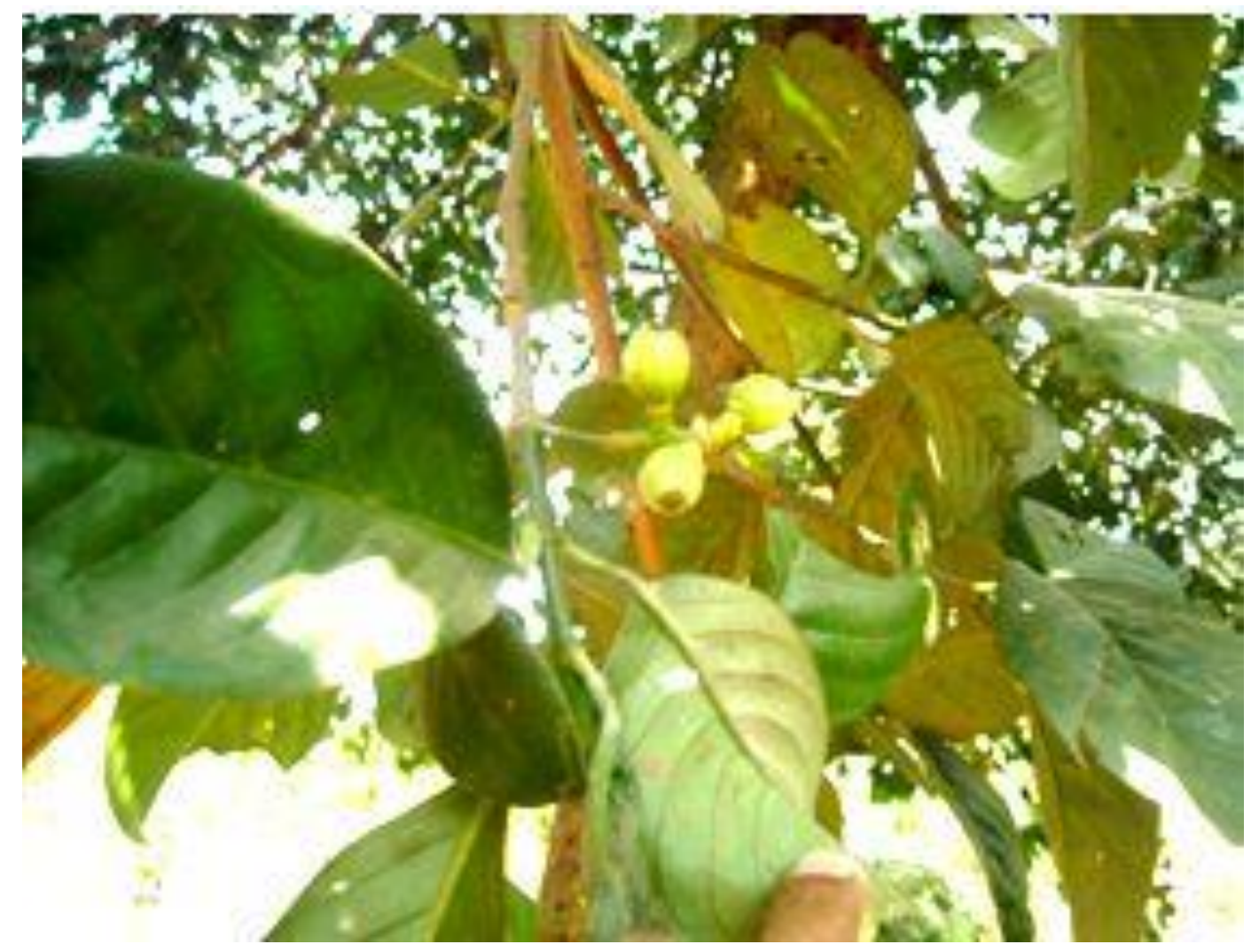

Fig. 10. Garcinia paniculata (Photo: Sanjib Baruah) 


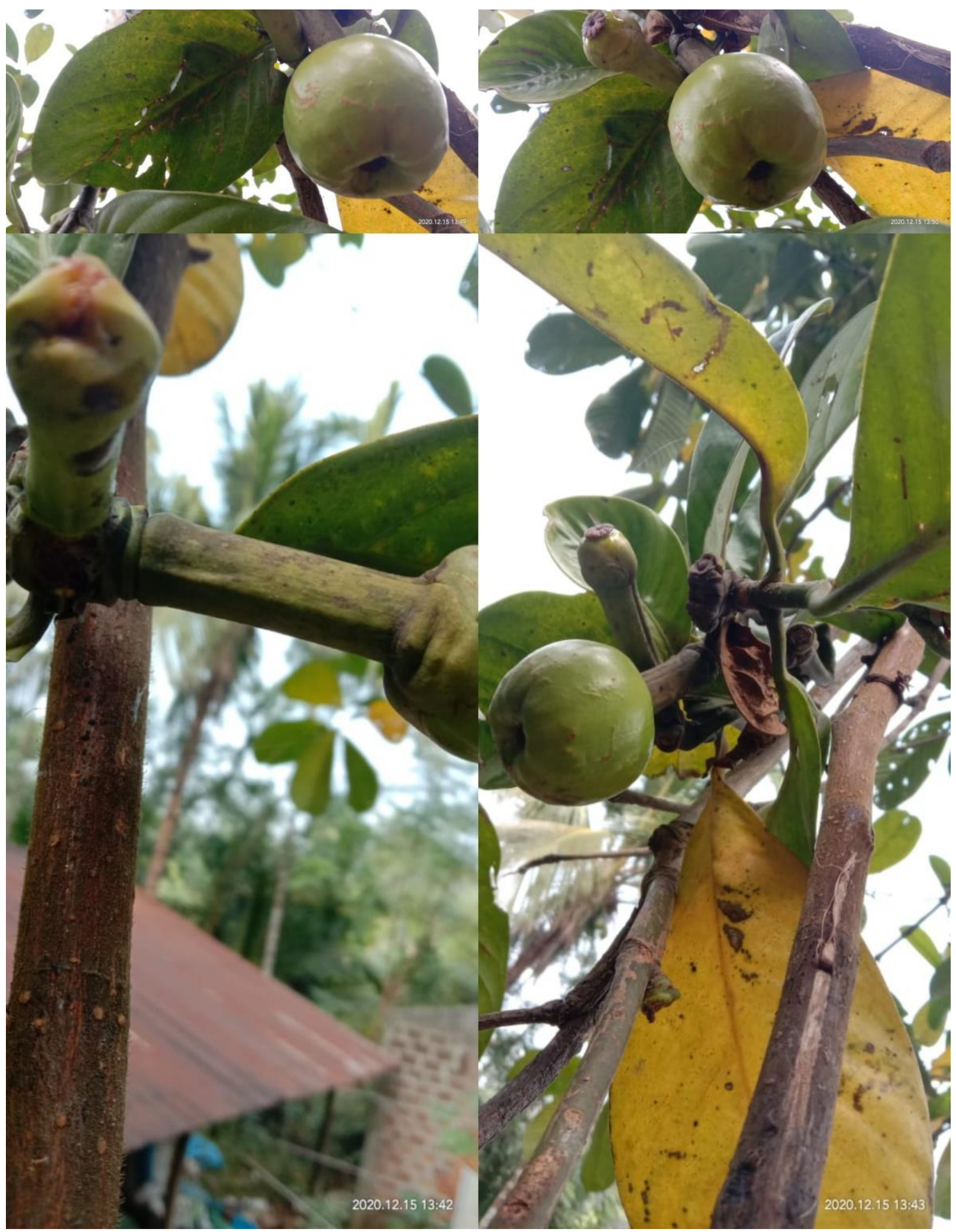

Fig. 11. Garcinia pedunculata (Photo: Sanjib Baruah) 


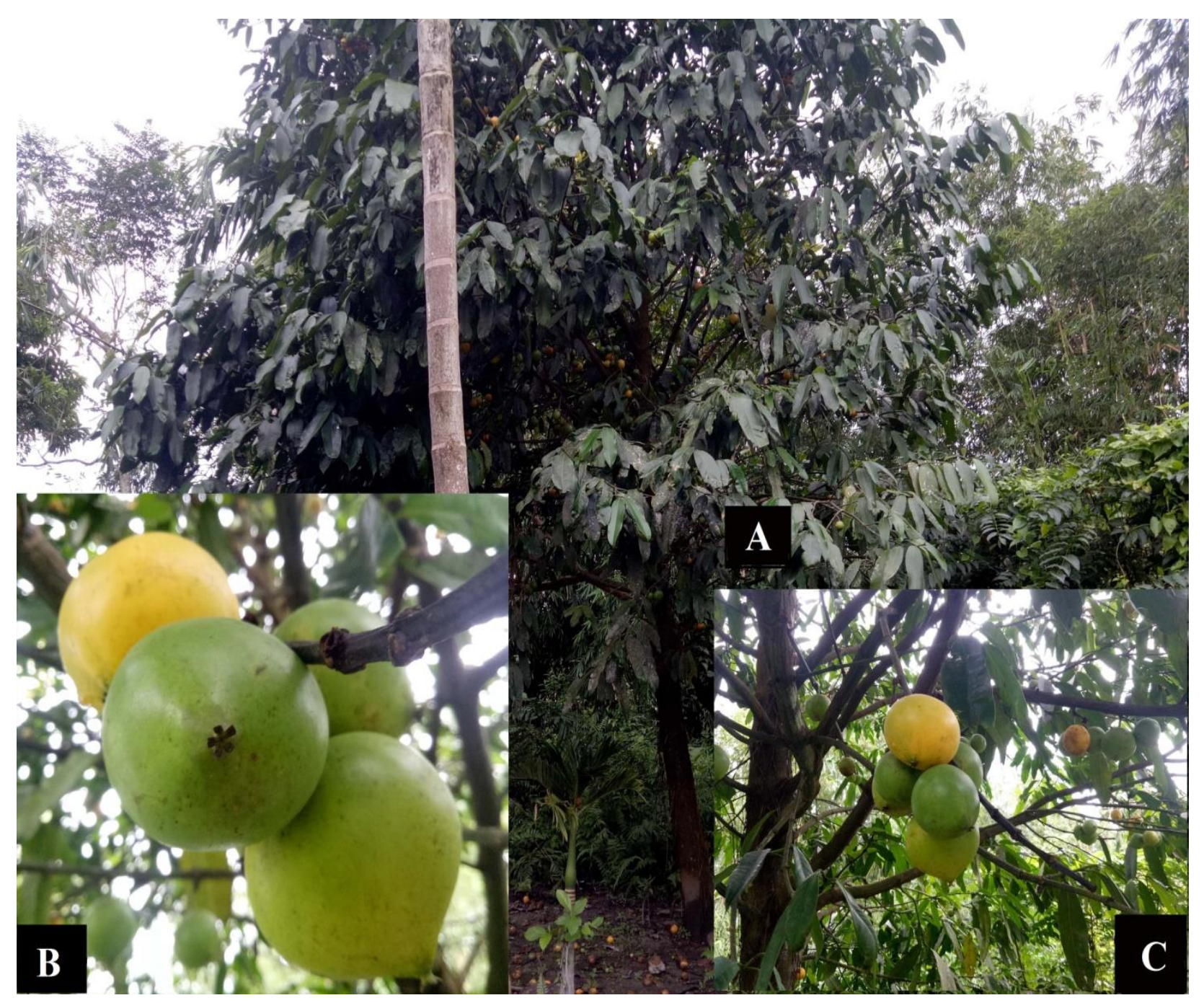

Fig. 12. Garcinia xanthocymus (A-A mature plant; B \& C-Mature fruits) (Photo: Sanswrang Basumatary)

\section{Garcinia morella (Gaertn.) Desr. (Figure 9)} Dioecious; middle sized evergreen trees, up to 15 $\mathrm{m}$ tall, wood moderately hard, bark dark brown, branchlets often opposite, latex thick resinous. Leaves simple, opposite, ovate-elliptic to obovate, ca. 10-15 × 4-10 cm across, base acute to cuneate, margins entire, midvein prominent; Flowers polygamous, tetramerous; male flowers: axillary, 3 flowered, ca. 5-8 mm across, sepals 4 , imbricate, petals 4 , imbricate, broadly elliptic, stamens 25-40. Female flowers: axillary, globose, with 10-12, staminodes, ovary superior, subglobose, 4 locular, Fruit fleshy berry, $1.5-2 \mathrm{~cm}$. in diameter, yellow when in ripe. Seeds 4-12, dark brown, juicy pulp.

Phenology:-Flowering February-March; Fruiting April-June

Distribution: India (Assam, Arunachal Pradesh, Meghalaya, Nagaland, Kerala), Sri Lanka.
9. Garcinia paniculata Roxb. ex Wight (Figure 10) Dioecious; small evergreen trees. Leaves simple, elliptic or oblanceolate acuminate, sub-coriaceous $11-15 \times 4.5-6.5 \mathrm{~cm}$., midvein stout prominent, lateral vein distinct. Petiole long 2.2-2.8 $\times$ 1.1-1.4 $\mathrm{cm}$. in size. Pedicels short $0.4-0.6 \times 1-1.2 \mathrm{~cm}$. in size. Flowers polygamous, 4-merous, male flower white sepals and petals 4 each, stamens numerous. Fruits small $3-41 \times 12-15 \mathrm{~cm}$ in size, cherry, yellow, succulent with granular stigma. Seeds generally 4 , enclosed in a pulpy aril.

Phenology:-Flowering during December-February; Fruiting March-April

Distribution: India (Assam, Meghalaya \& Nagaland).

\section{Garcinia pedunculata Roxb. Ex Buch-Ham.} (Figure 11)

Dioecious; large evergreen trees, rather short spreading branches. Leaves $13-30 \times 15-21 \mathrm{~cm}$. 
obovate or oblanceolate, rigid sub-coriaceous. Midvein stout prominent, lateral vein distinct. Petiole long 1.5-2.5 $\times 1.2-1.3 \mathrm{~cm}$. Pedicels long and thickened, 3-5 × 4-4.5 cm. Flowers polygamous, 4merous; male flowers large pale green, stamens many. Fruits large, become yellowish when ripe. Mature fruits $10-12 \mathrm{~cm}$. in diameter. Fresh wt. of the mature fruits av. 500-600 gms. Seeds 4-8 per fruits, enclosed in a fleshy or succulent aril.

Phenology:-Flowering January-March; \& Fruiting November-May

Distribution: India (Assam, Arunachal Pradesh, Meghalaya \& Nagaland).

\section{Garcinia nervosa Miq.}

Dioecious; evergreen middle sized trees, 7 to $12 \mathrm{~m}$ tall; young branches stout, compressed, 4-angled, 2 of the angles winged. Leaves simple, opposite large, $22-50 \times 8.9-17.8 \mathrm{~cm}$, lanceolate to oblong, petiole $3.4 \mathrm{~cm}$. Male flowers: not seen. Female flowers grow in cluster, creamy-white about $2 \mathrm{~cm}$. in diam., in axillary fascicles of $8-10 ; 2.5-3.5 \mathrm{~cm}$ long. Sepals 5, unequal, orbicular, much imbricate and very concave. Petals 5 , much larger than the sepals, orbicular. Disk of 5 thick, fleshy, pitted glands with 5 minute staminodes between them each bearing 4-5 minute imperfect anthers. Ovary ovoid, 5-rayed style, 5-celled. Fruit ovoid, yellow with red blotches when ripe, $5-5.2 \mathrm{~cm}$ long, with a mammilla, stigma distinct, 5-lobed. Seeds 2, elongated ovoid.

Phenology:-Flowering March-August; Fruiting April-October
Distribution: India (Assam, Meghalaya and Nagaland), Thailand, Peninsular Malaysia, Philippines, Singapore.

\section{Garcinia xanthochymus Hook. f. ex}

T.Anderson (Figure 12)

Dioecious; evergreen middle size trees, up to 15-20 $\mathrm{m}$ tall, bark dark brown, branches opposite, drooping Leaves simple, opposite, $25-32 \times 4-8 \mathrm{~cm}$., narrowly oblong or oblong lanceolate subcoriaceous to leathery shining in both surfaces, base cuneate to acute, apex acute to acuminate; midvein prominent, lateral vein distinct; petiole thick, stout. Pedicels short and thickened. Inflorescence axillary, cymes and fascicled; flowers polygamous, pentamerous, creamy-white coloured; male and female flowers borne on the axils of the fallen leaves. Sepals -5 , petals -5 , stamens -5 . Stigma oblique. Fruits oblong, sub-globose, about $3.5-6 \mathrm{~cm}$. in diameter, yellow in colour when ripe. Seeds 2-6.

Phenology:-Flowering March-May; Fruiting October-February

Distribution: India (Assam, Meghalaya, Western Ghats and Andaman islands).

\section{Ethnobotany}

A total of 12 species were found to be traditionally used by the different ethnic communities of Assam. Ethnobotanical uses, local names and ethnobotanical applications were recorded from interviewees of Assamese, Bodo, Karbi, Garo and other ethnic groups (Table 1).

Table 1 - Diversity and Ethnobotanical knowledge of Garcinia plants in Assam, Eastern Himalayas

\begin{tabular}{|c|c|c|c|c|c|}
\hline Taxa & Ethnic names & $\begin{array}{l}\text { Altitudinal } \\
\text { range }(\mathbf{m})\end{array}$ & $\begin{array}{l}\text { Part } \\
\text { used }\end{array}$ & $\begin{array}{l}\text { IUCN } \\
\text { Status }\end{array}$ & Ethnobotanical uses \\
\hline $\begin{array}{l}\text { Garcinia anomala } \\
\text { Planch. \& Triana }\end{array}$ & $\begin{array}{l}\text { Kujithekera } \\
\text { (Assamese) } \\
\text { Prangso-arong } \\
\text { (Karbi) }\end{array}$ & $\begin{array}{l}900-1800 \\
m\end{array}$ & $\begin{array}{l}\text { Fr, L, } \\
\text { Wo }\end{array}$ & NE & $\begin{array}{l}\text { The fruits are edible, consumed } \\
\text { by the Assamese community. } \\
\text { Mature wood is used in } \\
\text { construction of house. Apart } \\
\text { from this, leaves are eaten as } \\
\text { vegetable by Karbi tribe of the } \\
\text { state. }\end{array}$ \\
\hline \begin{tabular}{l}
\multicolumn{2}{l}{ Garcinia assamica } \\
J. \\
Shameer \& N.
\end{tabular} & $\begin{array}{l}\text { Thekera, } \\
\text { Kujithekera } \\
\text { (Assamese) }\end{array}$ & $100-300 \mathrm{~m}$ & $\mathrm{Fr}$ & $\begin{array}{l}\text { DD } \\
\text { (Sharma et } \\
\text { al. 2016) }\end{array}$ & $\begin{array}{l}\text { The pericarps of fruits are } \\
\text { edible and used for making } \\
\text { pickles by locals in Assam. }\end{array}$ \\
\hline
\end{tabular}

Mohanan

Garcinia cowa Cow-Thekera $100-500 \mathrm{~m} \quad \mathrm{Fr}, \mathrm{L} \quad \mathrm{NE} \quad$ Fruits eaten raw. Sun-dried

Roxb. ex Choisy (Assamese) slices are preserved in Assamese household. Fruits and leaves used in dysentery, diarrhea. Leaves are eaten by hill people of Assam. Fruits also used in headache.

\begin{tabular}{llllll}
\hline $\begin{array}{l}\text { Garcinia } \\
\text { dulcis (Roxb.) } \\
\text { Kurz }\end{array}$ & $\begin{array}{l}\text { Thekera } \\
\text { (Assamese) }\end{array}$ & $100-900 \mathrm{~m}$ & $\mathrm{Fr}$ & $\mathrm{NE}$ & Fruits are eaten raw. \\
\hline $\begin{array}{l}\text { Garcinia indica } \\
\text { (Thouars) Choisy }\end{array}$ & $\begin{array}{l}\text { Prangso-arong } \\
\text { (Karbi); } \\
\text { Thekera }\end{array}$ & $50-550 \mathrm{~m}$ & $\mathrm{Fr}$ & $\begin{array}{l}\text { Endemic to } \\
\text { India } \\
\text { (Sharma \& }\end{array}$ & $\begin{array}{l}\text { Fruits eaten raw; used to } \\
\text { remedy gastric flity, flatulence, constipation } \\
\text { aroblems: }\end{array}$ \\
\hline
\end{tabular}




\begin{tabular}{lc}
\hline (Assamese) & Sanjappa \\
$1993)$
\end{tabular}

and indigestion. Juice is a healthier and refreshing drink in summer. Sun dried fruits (kokum)used in curry. Seeds used for burning sensation and pain. Kokum consumed as sherbat in the western Ghats and in other parts of India (Chate et al. 2019).

\begin{tabular}{|c|c|c|c|c|c|c|}
\hline $\begin{array}{l}\text { Garcia } \\
\text { Roxb. }\end{array}$ & kydia & $\begin{array}{l}\text { Chopchopa } \\
\text { (Assamese) }\end{array}$ & $200-1000 m$ & $\mathrm{Fr}$ & $\mathrm{NE}$ & $\begin{array}{l}\text { Ripe fruit pulps eaten raw. Dried } \\
\text { sliced pericarps of fruits used as } \\
\text { medicine for stomach disorders. }\end{array}$ \\
\hline
\end{tabular}

$\begin{array}{llllll}\text { Garcinia } & \text { Rupohithekera } & 100-1000 m & \text { L, Fr } & \text { NE } & \text { Fruit pericarp eaten raw. Dry }\end{array}$

lanceifolia Roxb. (Assamese) sliced pericarps made into fish
curry; also used for dysentery. Gamboge, the gum resin is used as medicine and as yellow dye. Fruit juice cures fever, jaundice and urinary troubles. Dry pericarp juice drunk as sherbet in summer. Leaves are taken as vegetables by the Assamese.

\begin{tabular}{lllllll}
\hline $\begin{array}{l}\text { Garcinia } \\
\text { lanceifolia }\end{array}$ var. Rupohithekera & & & Assamese. \\
\hline
\end{tabular}

\begin{tabular}{llllll} 
oxyphylla & (Assamese) & & & & \\
\hline Garcinia morella & Kuji-thekera & $100-$ & 1100 & $\mathrm{Fr}$ & $\mathrm{NE}$ \\
(Gaertn.) Desr. & (Assamese) & $\mathrm{m}$ & & &
\end{tabular}

(Assamese)

\begin{tabular}{|c|c|c|c|c|}
\hline $\begin{array}{l}\text { Garcinia } \\
\text { paniculata } \\
\text { ex Wight }\end{array}$ & Roxb. & $\begin{array}{l}\text { Sosopatenga } \\
\text { (Assamese) } \\
\text { Marlo (Karbi) }\end{array}$ & $\begin{array}{l}100-1000 \\
m\end{array}$ & Fr, L, W \\
\hline
\end{tabular}

\begin{tabular}{lllll}
\hline Garcinia & Borthekera & $100-1000$ & Fr, Wo & NE \\
pedunculata & (Assamese) & $\mathrm{m}$ & \\
Roxb. Ex Buch- & & & \\
Ham. & & & \\
\end{tabular}
occurs in yellow colour; oil and juice of fruits treat fever, diabetes and jaundice.

Fruits are eaten raw or dried; cures dysentery. A commercial source of 'gamboge', that Leaves used to treat roundworm. Moderately hard word used for house construction and firewood.

Ripe fruits are acidic and eaten raw. Fruits sliced into small pieces, and dried under sun for 10-12 days for preservation. Dried pericarps of fruits treat dysentery, digestive and cooling. Fruits also used as fixative or as a mordant for saffron dye. Wood used for making house, furniture and traditional rice mill "Dheki" (Baruah \& Borthakur 2012).

\begin{tabular}{|c|c|c|c|c|c|}
\hline $\begin{array}{l}\text { Garcinia nervosa } \\
\text { Miq. }\end{array}$ & $\begin{array}{l}\text { Thekera } \\
\text { (Assamese) }\end{array}$ & $50-500 \mathrm{~m}$ & Fr, L & $\mathrm{NE}$ & $\begin{array}{l}\text { Fruit pulp is edible, sour in } \\
\text { taste. Ripe fruits cure } \\
\text { dysentery; leaves used as cattle } \\
\text { laxative. }\end{array}$ \\
\hline $\begin{array}{l}\text { Garcinia } \\
\text { xanthocymus } \\
\text { Hook.f. } \\
\text { T.Anderson. ex }\end{array}$ & $\begin{array}{l}\text { Teportenga } \\
\text { (Assamese) }\end{array}$ & $50-500 \mathrm{~m}$ & $\mathrm{Fr}, \mathrm{B}$ & $\mathrm{NE}$ & $\begin{array}{l}\text { Fruits are acidic and eaten raw. } \\
\text { Ripe fruits used for making } \\
\text { jams, delicious chutney and } \\
\text { curry by the Assamese. A } \\
\text { sherbet from dried fruits is given } \\
\text { in dysentery. Fruit latex is } \\
\text { medicine for curing wound and } \\
\text { skin diseases. Bark of the tree } \\
\text { and latex of unripe fruits are } \\
\text { used to make yellow dye. Wood } \\
\text { is hard, good for making house. }\end{array}$ \\
\hline
\end{tabular}




\section{Discussion}

The genus Garcinia includes members that are mostly small to medium-sized trees, which are important component of tropical forests of Assam. Of the 12 species, 5 species viz., Garcinia pedunculata, G. lanceifolia, G. cowa, G. xanthocymus, and $G$. morella have relatively wider geographic distribution, and are used by local people very often. Three species are not so common and have a restricted geographical distribution: Garcinia anomala Roxb. G. kydia and G. assamica. According to Anderson (1874) and Maheshwari (1964), G. xanthochymus is distributed in the Western Ghats. However, scrutiny of literature and field collections revealed that $G$. xanthochymus is naturally located only in the Northeast India including plains of Assam. The study revealed that majority of the Garcinia species has not been assessed properly (Table 1).

The study revealed all Garcinia species can be arranged in different categories on the basis of traditional uses (Table 1). Among the categories, the most frequent are edible fruits, followed by ethno medicinal uses, and wood. A few Garcinia species are cultivated either for fruits, traditional medicines or other domestic uses such as for making house, firewood and landscaping. Among the cultivated species are included Garcinia pedunculata, G. cowa, G. morella, G. lanceifolia and $G$. xanthocymus. Members of Garcinia L. species produced edible fruits and $G$. pedunculata is often considered as most famous fruit in Assam. The young leaves of the few members of Garcinia (G. anomala, G. pedunculata and G. paniculata) are eaten cooked by some tribes in the northeast region (Arora 1981, Jain \& Dam 1979, Rao et al. 1981). The latex of $G$. cowa is used in Thai folk medicines as an antifever agent (Pattalung et al. 1994). Members of Garcinia L. used after childbirth medication, for menstrual problems, dysentery and fever in traditional system of medicine. It is observed that sun-dried slices of the fruits are used for culinary purposes and as folk medicine by the indigenous community of the state. Some species like G. xanthocymus, G. indica, and G. cowa are cultivated in certain parts of India. G. pedunculata, G. kydia, G. cowa, and G. lanceifolia are the most important species in northeastern parts of India, particularly in the study area. Many species of Garcinia have fruit with edible arils and are eaten locally (Baruah \& Borthakur 2012).

\section{Conclusions}

The present survey revealed that a handsome number of Garcinia species occur in the state. The results obtained indicate that the use of Garcinia species is very widespread in the region has confirmed a total of 12 species and 1 variety. Literature review revealed that Garcinia species are found to be potential sources of diverse secondary metabolites. However, commercial cultivation and market value are still unknown to the rural community. The study proves that though the region has rich diversity of Garcinia species, it has not been studied properly. This study concluded that documentation and preservation of ethnobotanical knowledge associated with Garcinia members is an utmost important which could generate further research activities for the benefits of humankind.

\section{Declarations}

List of abbreviations: L: Leaves, FL: Flower, B: Barks, Fr: Fruits, $\mathrm{m}$ asl: meter above sea level, $\mathrm{m}$ : meter, Fr: Fruits, W: Wax, Wo; Wood, NE: Not Evaluated, DD: Data Deficient

Ethics approval and consent to participate: Consent was obtained from all participants before conducting interviews.

Competing interests: The authors declare that they have no competing interests.

Availability of data and materials: All relevant data are available within the manuscript.

Funding: This research work is self-funded and didn't receive any grant from funding agencies.

Authors' contributions: SB, PB designed this study; SB collected and analyzed data and wrote the initial draft of the manuscript. SB, PB revised and edited the manuscript. SWB, BB did the preliminary work, interviews, plant collection, and data generation.

\section{Acknowledgements}

The authors would like to acknowledge the local communities for sharing their knowledge and kind cooperation during field visits. The first author gratefully acknowledges Mr. Jatindra Sarma, IFS, dynamic forest officer of Assam for his contribution by providing some of the images.

\section{Literature Cited}

Anderson T. 1874. Guttiferae. In Flora of British India. Edited by JD Hooker 1. L. Reeve and Co., London.

Arora RK. 1981. Native food plants of the northeastern tribals, In Glimpses of Indian Ethnobotany. Edited by SK Jain. Oxford \& IBH Publication, New Delhi, Pp. 91-106.

Barooah C, Sarma L. 2016. Vertebrates of Assam; a checklist with IUCN status. Assam Science Technology and Environment Council, Guwahati, Assam. 
Baruah S, Borthakur SK, Gogoi P, Ahmed M. 2011. New distributional record of Smilax china Linnaeus in India. Pleione 5(2): 325-327.

Baruah S, Sarma J, Roy H, Borthakur SK. 2013. Notes on two interesting Angiosperms of Assam, India. Pleione 7(2): 401-405.

Baruah S, Borthakur SK. 2012. Studies on morphological and ethno-botany of six species of Garcinia L. (Clusiaceae) found in the Brahmaputra valley, Assam. Indian Scholars Research Library 2(3): 389-396.

Baruah S, Gogoi P, Ahmed M, Borthakur SK. 2012. Traditional Knowledge of Dental Care plants in Assam. Advances in Plant Science 25(2): 394-399.

Baruah S, Brahma D, Upadhya P. 2018. Phytochemical study of some selected medicinal plants and its ethnobotanical importance to the indigenous communities of Assam. Medicinal Plants 10(2):145-150.

Begam A, Borthakur SK, Sarma J. 2013. Garcinia dulcis (Roxburgh) Kurz (Cluciaceae): a new distributional record for Assam, India. Pleione 7(2): 545-548.

Chate MR, Kakade SB, Neeha VS. 2019. Kokum (Garcinia indica) Fruit: A Review. Asian Journal of Dairy and Food Research 38(4): 329-332.

Chowdhury S. 2005. Assam's Flora Present Status of Vascular Plants. Assam Science Technology and Environment Council, Guwahati, Assam.

Dutta B, Borborah K, Borthakur SK. 2014. Garcinia nervosa miq. a new record from mainland India. Indian Journal of Plant Sciences 4(3): 34 -36.

Jain SK, Dam N. 1979. Some ethnobotanical notes from north-eastern India. Economic Botany 33: 5256.

Jain SK, Rao RR. 1977. A hand book of Field and Herbarium Technique. Today and Tomorrow's Printers and Publishers, New Delhi.

Jones S. 1980. Morphology and major taxonomy of Garcinia (Guttiferae). Ph.D. dissertation. London, University of Leicester and British Museum.

Kanjilal UN, Kanjilal PC, Das A. 1934. Flora of Assam. Periodical Expert Book Agency. Delhi.

Kar A, Borkakoti S, Borthakur SK. 2008. Extended distribution of the genus Garcinia L. in Sonitpur district, Assam, India. Pleione 2(2): 165-170.

Mabberley DJ. 2005. The plant Book- A Portable dictionary of the vascular plants (2nd ED), Cambridge University Press.
Maheshwari JK. 1964. Taxonomic studies on Indian Guttiferae III. The genus Garcinia L.s.I. Bulletin of the Botanical Survey of India 6(2/4): 107-135.

Mohanan N, Shaju T, Rajkumar G, Pandurangan AG. 1997. Rediscovery of Garcinia imberti Bourd. (Clusiaceae), a little known endemic species of Western Ghats. Indian Journal Forestry 20 (4): 383385.

Nimanthika WJ, Kaththriarachchi HS. 2010. Systematics of genus Garcinia L. (Clusiaceae) in Sri Lanka. New insights from vegetative morphology. Journal of National Science Foundation 38: 29-44.

Pattalung $\mathrm{P}$, Thongtheeraparp $\mathrm{W}$, Wiriyachitra, $\mathrm{P}$, Taylor WC. 1994. Xanthones of Garcinia cowa. Planta Medica 60(04): 365-368.

Rao MK, Shanpru R. 1981. Some plants in the life of the Garos of Meghalaya. In Glimpses of Indian Ethnobotany. Edited by SK Jain, Oxford \& IBH Publication, New Delhi, Pp. 153-160.

Sabu T, Mohanan N, Krishnaraj MV, Shareef SM, Shameer PS, Roy PE. 2013. Garcinia pushpangadaniana, (Clusiaceae) a new species from southern Western Ghats, India. Phytotaxa 116 (2): 51-56.

Sarma J, Baruah S, Borthakur SK. 2014. New distributional record of Garcinia indica (Thouars) Choisy for Assam as well as the North-Eastern part of India. Journal of Economic and Taxonomic Botany 38: 121-124.

Sarma J, Shameer PS, Mohanan NN. 2016. A new species of Garcinia (Clusiaceae) from Assam, North East India. Phytotaxa 252(1):73-76.

Singh NP.1993. Clusiaceae. In Flora of India Vol. 3. Edited by BD Sharma, M. Sanjappa. Botanical Survey of India Calcutta Pp.86-151.

Srivastava SK. 1994. Garcinia dhanikhariensis (Clusiaceae), a new species from Andaman Islands, India. Nordic Journal of Botany 14: 51-53.

Stevens PF. 2007. Clusiaceae. In The families and genera of vascular plants. Edited by $\mathrm{K}$ Kubitzki. Springer, Berlin.

Thiers B. 2018. Continuously updated. A Global Directory of Public Herbaria and Associated Staff, New York Botanical Garden's Virtual Herbarium.

Whitmore TC. 1973. Guttiferae. In Tree Flora of Malaya, a Manual for Foresters. 2, Edited by TC Whitmore. Longman, London. 